\title{
The Need of College English Ability
}

\author{
Weina Ouyang, ${ }^{1, a}$ and Xue Han ${ }^{1, b^{*}}$ \\ ${ }^{1}$ College of Foreign Language Education, Beihua University, Jilin City, Jilin Province, 132000, China \\ *the corresponding author
}

Keywords: English ability; Social needs; In vestigation and research

\begin{abstract}
For a long time, college English education in our country is under a serious imbalance with a huge investment and limited output. The current situation of college students' English ability is at a low level and does not match the needs of social employers. Therefore, the problem of demand and structure of college student' English ability has become a social topic of great concern. Through investigating and studying the current situation of undergraduates' English in our country, the paper explores the system of cultivating English ability of undergraduates, emphasizes the combination of the humanity and instrumentality in undergraduates' English, and highlights the important role of English tool attributes in meeting the needs of society. Finally, this paper puts forward the methods to improve college students' English ability, to improve teaching plan, to supplement special English learning and grasp social needs in time so as to provide a new perspective for college students' English ability cultivation.
\end{abstract}

\section{大学生英语能力需求 \\ 欧阳嵬娜，韩雪 \\ 北华大学公共外语教育学院, 吉林吉林 132000}

摘要: 长期以来, 我国大学英语教育都处在投入巨大、产出有限的严重失衡状态之下, 大学生的英语能力现状整体处在 较低的水平上, 与社会用人单位的需求不相匹配, 因此, 大学生英语能力的需求与结构问题已经成为一个备受关注的社会性 话题。通过对我国大学生英语现状的调查研究, 对大学生英语能力培养的体系进行了探讨, 强调了大学英语人文性和工具性 的结合, 并突出了英语的工具属性在满足社会需求方面的重要作用, 最后提出了提升大学生英语能力的方法, 完善教学计划、 补充特殊英语学习、及时把握社会需求等, 以期为当下大学生英语能力培养提供全新视角。

关键词: 英语能力; 社会需求; 调查研究

中图分类号：H319 文献标志码： A

\section{1 引言}

改革开放以来, 我国的教育水平有了长足的进步, 但是英语教育却长期受到脈病, 投入了大量的时间 和财力, 但却收效甚微, 没有得到普遍的提升。义务教育的学生从小学开始就接触英语, 直到进入大学通 过四六级专业考试, 长达数十年, 但依然没有办法流利的用英语进行交流和对话, 这是一个不容回避的问 题。造成这种问题出现的原因肯定是多方面的, 其中考试作为一个重要的环节, 也必须承担必要的责任。 因此, 这就引发了对于大学生究竟需要怎样的英语能力的讨论和思考, 对大学生英语能力需求的探究就变 得很有必要 ${ }^{[1]}$ 。

\section{2 目前我国大学生英语能力水平现状}

\section{1 大学生英语能力并不乐观}


我国在刚开始推行英语四级考试时, 对大学新生的词汇量要求为 1600 个, 这个要求相比起大学四六 级考试的要求普遍较低, 这对于大学生努力学习英语动力较大, 不过随着我国教育条件和环境的逐渐改善, 很多学生在高中毕业的时候就已经掌握了 3500 个以上的词汇, 基本已经达到了大学英语四级的要求, 整 体对英语的掌握程度也有了比较大的提升, 加之我国现行的大学英语教材还是以考试为最终目的, 内容以 及体系方面都和中学的英语教材有着很多的重复, 熟悉的内容直接导致了学生学习的热情和积极性下降; 此外, 鉴于我国东西部教学设施和教学质量的较大差距, 造成普通的本科院校需要分成几个批次进行录取, 相应的学生水平也是层次不齐, 如果采取一刀切的方式, 以同一标准对学生英语能力进行考量, 带给教学 的难度也会很大, 这也使得每个高校很难根据实际情况进行个性化教学大纲的难度变大。根据相关的调查 显示, 2010 年 6 月全国 16 所本科院校 1200 余名大学生中, 有 $25.5 \%$ 的学生认为自其入学以来英语水平“没 有明显提升”, 35. 1\%的学生认为自己的英语能力反而有些退步, 不如高中时期。

虽然比起上世纪八十年代, 现在的大学新生英语能力普遍提升不少, 但与历史其它时期进行比较, 比 如民国时期, 就会显得有些落后。数据显示, 在民国时期, 1932 年对高中生的英语词汇量要求是 8000 个, 其中包括 5300 个复用词汇, 在那个时期, 我国大学新生的英语水平保持在世界先进水平, 横向比较, 要 比日本以及俄罗斯的新生要求标准要高。这些不同时期的不同要求也导致了我国大学新生在一入学时英语 能力就各不相同。

此外, 有学者研究指出, 从现阶段我国大学英语的能力要求来看, 大学英语还是被作为一门基础课程, 主要以语言技能的掌握和实际的应用的综合运用为主, 在教学过程中偏重其工具属性, 强调技能培训。在 英语内容教学中, 人文性主题较多而专业属性主题较少, 英语教材结构的不合理也在很大程度上制约了我 国大学生英语综合能力的全面均衡发展 ${ }^{[2]}$ 。

通过上述内容可以看出, 我国大学生目前的英语能力整体偏弱, 而且发展不平衡, 现状堪忧。

\section{2 大学生英语能力欠缺科学合理的评价机制}

当前在我国, 很多高校仍然把考试作为主线, 将大学英语四六级考试的成绩作为评价办学实力的硬性 指标, 这直接导致了诸多高校英语教学目标单一, 只抓住四六级考试成绩和通过率不放, 有的高校领导甚 至将四六级成绩当成自己政绩进行展示, 对英语四六级成绩的过度重视导致了学校教学精力和财力分配的 严重不平衡, 其它学科的教学资源和教学时间被严重挤压, 有些高校甚至被讽刺为 “伪外国语大学”, 说 明这一现象已经到了非常严重的程度。

此外, 我国英语教育同质化的问题也已经非常严重, 表现在英语教育中投入产出比较低, “费时低效” 情况很普遍, 许多高校的英语教学只是中学英语的 “夹生饭”, 大学中学习的内容很大程度上是中学内容 的重复, 包括基本的语法以及修辞等。有学者也对这种现象提出了尖锐的批评, “高校英语教学只能提升 考试分数, 对语言能力提升毫无作用”; “我国每年都有许多的高校学生进行四六级考试, 虽然很多都能 顺利通过, 但到了实际使用时, 无法开口、不能下笔的现象普遍存在, 这充分暴露了我国英语教学体系的 缺陷” ; “国内流行的四六级考试并不是综合的能力测试, 达到了合格线却并不能表示实际已经具有了某 种能力” ${ }^{[3]}$ 。

调查研究的结果也证实了这一观点, 在对如何看待大学英语四六级考试方面, 56. 9\%的被调查者持负 面的看法, $44.5 \%$ 的被调查者持正面看法, 仅有 $9.2 \%$ 持完全肯定的态度; 另一调查显示, 尽管 $90 \%$ 的被调 
查者均受过外语教育, $70 \%$ 甚至学习长达六年的时间, 但仍有高达 $64.4 \%$ 的被调查者完全不能运用英语交流 或者仅能勉强说上几句, 能熟练进行交际的仅占 $5 \%$, 表明我国现阶段英语教育目标较低, 不能够满足经济 社会发展的要求 ${ }^{[4]}$ 。

综上所述, 我国目前还没有建立起统一的英语语言能力衡量体系, 对英语教学的目标缺乏统一标准, 这样既不利于确定实际的教学目标, 也不利于相关课程的深入, 这一问题应当引起高校乃至社会的高度关 注 $^{[5]}$ 。

\section{3 我国大学生英语能力培养体系探讨}

针对目前我国大学生英语并不乐观的现状以及社会对大学生英语能力的迫切需求, 大学生英语能力的 制定标准就应当不拘一格, 尽可能的体现多样化以及差异化的需求。目前, 已经有学者提出了相关的理论, 其中比较重要的是从通用英语的教学转向学术英语教学并且建立起学术英语能力的不同层级。但根据我国 现状, 研究性大学还是少数, 另外随着高校扩招, 本科教育也趋于大众化, 未来众多的大学毕业生从事学 术性工作的比例也会逐渐较小, 社会对旅游英语、商务英语以及行政英语等的需求会越来越强烈, 因此由 通用型向专业型的转变未免相对片面。

因此，在我国，应当依据高校、专业情况、不同地区、学生个人等等的实际情况，制定不同的能力标 准, 由学生自主决定是否要达到其中标准之一, 在这一方面已经有一些有益的尝试。目前广泛流行的特殊 用途英语已经体现了这一方面的尝试。特殊用途英语更加注重英语的工具性以及实用性, 能够帮助大学生 自主明确适合自己能力的标准。特殊用途英语课程的目标以及内容的制定, 通常不会根据常规的通识教育 来设定, 而是根据功利性、实际性以及需求性的学习要求来确定。特殊用途英语的流行, 表明大学英语除 了具有人文性的特点外, 其工具属性也不容忽视, 已经成为满足社会需求的重要部分。

此外, 有学者研究认为, 目前我国已经成为英语学习的大国之一, 我们学习英语的最终目的, 包括 “向 己型” 以及 “向他型” 这两种。“向己型” 是指通过对英语的学习进而学习外国先进的科技以及管理经验; “向他型” 则是针对更加广泛的其它语言的学习服务而言。随着我国经济社会的不断发展, 国际地位的不 断提高, 我国外语学习中 “向他型” 趋势明显, 在未来也会变得越来越重要。

理论研究必须要经过实践的检验, 这在英语学习上也是如此。根据相关的访谈以及调查数据, 显示社 会用人单位以及大学生自身均对英语的人文属性和工具属性有着清醒的认识, 二者都是英语能力中不可或 缺的重要部分。社会用人单位更加实际一些, 对于大学生英语人文方面的能力不做过多要求, 但对工具方 面的能力有较高要求, 希望大学生在毕业之后都能够将英语运用到极致; 而对于大学生自身而言, 其对于 人文能力的要求要高于用人单位, 根源是大学生经过系统的教育, 迫切希望自己在各个方面都能够全面发 展, 而不想仅仅成为工具的存在, 而且通过对人文能力的追求可以使大学生在工作之后能够得到更好的成 长。针对目前大学生英语能力能够胜任工作需求的调查显示, 半数以上的用人单位均持否定意见, 认为大 学生存在 “好高鹗远” 的情况, 此外, 大学生对自己的英语能力水平表示并不满意, 但对于用人单位的担 忧表示可以理解。针对大学英语教学的调查, 表明用人单位和大学生均认为现行的教学内容与实际应用脱 节严重, 应当根据社会需求以及大学生未来的职业发展, 制定更具有针对性的教学计划, 要注重特殊用途 英语的教学 ${ }^{[6]}$ 。

\section{4 我国大学生英语能力需求研究}


当前, 高校大学生的英语能力主要由人文性能力以及工具性能力两大部分组成, 二者互相补充, 缺一 不可。新的修订后的英语课程教学要求也对大学英语的性质进行了阐述和说明 “大学英语不仅仅是一门基 础的语言课程, 它同时也是学生开拓眼界、了解世界的一个窗口, 兼有人文和工具两个方面的属性” , 只 有这两个方面全面发展, 大学生才能够养成现代社会的基本素质以及特征。伴随着经济社会的过快发展, 社会上很多人将主要的注意力都集中到了对金钱等物质利益的追求上, 放松了对自己精神方面的要求, 道 德水平下降明显, 这也直接导致了大学生人文素养等的降低。大学英语人文能力的长期欠缺, 最终也会对 其工具属性产生不利的影响。丰富并且完善的人文属性应当是语言学科的重要属性, 其对于提升学生道德 水平、人文素养的提升具有重要作用, 也是语言教学中所要传递的重要内容之一。

相对于人文能力, 工具能力也应当成为大学生能力养成的重要部分。英语虽然是一门广泛开展的学科, 但其培养目标应当具有针对性, 不能一刀切, 造成千人一面的现象。相应的, 大学生也应当根据自己的专 业、需求等实际情况, 选择特殊用途英语进行重点的学习。大学英语工具能力的培养, 不仅能够满足学生 的个性需求, 同时也为大学生未来的发展提供了良好的保障。

目前, 大学生英语能力的现状与社会需求之间呈现出以下三个方面的特点:

首先，现阶段大学生虽然英语学习年限很长，但实际应用起来仍然达不到社会需求，听、说、读、写 等各方面的单项能力短板明显, 尤其是口语的表达能力, 很多大学生甚至还停留在 “哑巴英语” 的水平或 者仅限于日常简单的打招呼等情况, 这种情况远远不能满足社会需求, 因此, 提升大学生英语工具属性的 能力, 锻炼英语表达能力至关重要, 相关的需求也比较强烈。

其次, 大学生自身对提升英语应用能力也有着极为迫切的需求, 很多大学生都能够清楚的意识到自身 所暴露出的能力缺陷和短板, 急于改变现状, 不满足于当前四六级考试的评价现状, 对提升社会需求的英 语听说等交流方面的能力渴望强烈。虽然在提升听说等能力方面需求巨大, 但也应当紧密结合社会需求, 跟随时代发展, 有针对性的进行补足。

第三, 社会对于大学生英语能力的需求不应当孤立的存在, 这种需求应当充分反应到大学生英语学习 的各个方面, 包括培养规划、课程设置、专业方向等方面。大学生也应当尽早的明确社会需求, 在大学英 语的日常学习中有计划的多加锻炼, 做到有备无患, 减少毕业之后转型的阵痛, 尽快与社会需求接轨, 更 快的融入到社会当中 ${ }^{[7]}$ 。

\section{5 结语}

任何一门语言的运动都包括用法以及应用两个层面, 用法主要解决的是对抽象知识的具体掌握, 而应 用则是指将学到的语言成功的运用于交际之中, 用法对应用的帮助是有限的, 而应用却可以大大加强对用 法的掌握, 从这个角度上讲, 对大学生英语能力的培养意义重大。本文主要分析了当前大学生英语能力的 现状, 对我国大学生英语能力培养的体系进行了探讨并进行了深入的研究, 表明大学生在英语的应用能 力方面函需加强, 需要运用完善教学计划、补充特殊英语学习、及时把握社会需求等方法进行加强, 以期 满足社会需求, 促进我国经济社会的快速发展 ${ }^{[8]}$ 。

\section{6 致谢}

基金项目: 本文系吉林省教育厅项目（项目名称：全媒体背景下大学生就业对英语能力需求研究; 项 目编号: JJKM20170089SK) 


\section{Acknowledgements:}

Fund project: This article is the project of the Education Department of Jilin Province(project name: "Study on the English ability demand of College Students' Employment under the background of omnimedia"; Project number: JJKM20170089SK)

\section{References}

[1] Z. Liu and D. M. Mei: Journal of Xi'an International Studies University, (2015) No. 3, p.65-68

[2] Y. Wang and W. Q. Wang: Computer-assisted Foreign Language Education, (2012) No. 3, p. 36-40

[3] B. C. Han: Foreign Language Teaching and Research, (2002) No. 6, p. 410-411

[4] Z. W. Lu and R. G. Zhang: A survey of China's Foreign Language Competence Requirements and Strategic Recommendations(Beijing University Press, Beijing2012)

[5] H. Z. Yang: Foreign Language Teaching and Research, (2012) No. 3, p. 292-297

[6] D. Zuo: A Study of the Relationship among English Teaching, English Skills and Job Demands (Changsha University of Science and Technology,2010)

[7] C. Guan, Y. B. Hou and Y. Li: Journal of World Education, (2009) No. 9, p. 36-38

[8] L. Zhu: University Education, (2013) No. 15, p. 13-14 